\title{
Binarity of Hipparcos Main Sequence Survey Stars
}

\author{
Carl F. Quist and Lennart Lindegren \\ Lund Observatory, Box 43, SE-22100 Lund, Sweden
}

\begin{abstract}
Hipparcos observations of double stars, mainly A and F primaries, are modelled in order to check the statistics of double-star solutions in the Hipparcos Catalogue and to set quantitative limits on binary distributions. The number of companions per primary is estimated to $0.25 \pm 0.05$ for semi-major axes from 1 to $10 \mathrm{AU}$, and to $0.43 \pm 0.20$ for the range 10-100 AU. The total multiplicity found is between 0.9 and 1.2.
\end{abstract}

\section{Introduction}

Stellar multiplicity, binary distributions (e.g. mass ratio and semi-major axis) and orbital evolution are important tracers of star formation and its environment. Here we focus on the semi-major axis distribution determined from observations of main sequence survey stars in the Hipparcos Catalogue (ESA 1997).

The study by Duquennoy \& Mayor (1991, hereafter DM) of solar-type field stars in the solar neighborhood is used as a standard model to which we compare our results. Their main results include: (1) a broad, approximately log-normal distribution of orbital periods with median value $P=180 \mathrm{yr}$; (2) a mass-ratio distribution continuously increasing towards small values of $q$; and (3) a total multiplicity of $57 \%$ (i.e., 0.57 companions per primary star). One shortcoming with surveys aiming at more distant stars has been the difficulty to study the multiplicity in the 1-10 AU separation range, the classical gap between the spectroscopic and visual techniques (Blaauw 1981). The Hipparcos satellite was however quite sensitive in the 1-10 AU range, thanks to the detection of numerous astrometric binaries, a property that is exploited in this study.

The Hipparcos survey was intended to give an unbiased sample of high precision astrometry for the brightest stars. The construction of the Hipparcos Input Catalogue (HIC, Turon et al. 1992) was based on the partially inaccurate or incomplete data available at that time, leading to a rather diffuse survey limit. We adopt a brighter limit, at the expense of the sample size, defined as

$$
V_{\text {tot }} \leq 7.0+1.1|\sin b|
$$

where $V_{\text {tot }}$ is the total magnitude of components within 10 arcsec of the primary. We also restrict our sample to main-sequence stars by means of a cut in parallax as function of $V$ and $B-V$. Dividing the resulting sample into spectral type, we find that $22 \%$ are $\mathrm{O}$ and $\mathrm{B}$ stars, $36 \% \mathrm{~A}, 33 \% \mathrm{~F}, 8 \% \mathrm{G}$ and $1 \% \mathrm{~K}$ and $\mathrm{M}$ stars. Further statistics can be seen in Table 1. 
Table 1. Statistics of the investigated Hipparcos Catalogue sample. The table gives the 5th, 50th and 95th percentiles of the quantities.

\begin{tabular}{lccc}
\hline quantity & $5 \%$ & $50 \%$ & $95 \%$ \\
\hline apparent magnitude, $V$ & 4.75 & 6.81 & 7.74 \\
colour index, $B-V$ & -0.12 & 0.23 & 0.64 \\
absolute magnitude, $M_{V}$ & -1.9 & 1.5 & 4.4 \\
observed parallax, $\pi$ [mas] & 2.4 & 9.2 & 36.6 \\
\hline
\end{tabular}

\section{Hipparcos Double Stars}

The Hipparcos Catalogue contains five types of binary solutions, to which we add a sixth $(\Delta \mu)$. Here, only a brief outline is given. Recall that the standard astrometric solution for a single star uses five parameters: position (2), proper motion (2), and trigonometric parallax (1). Binary solutions require additional parameters.

Component Solutions $(C)$ : The ' $C$ ' solutions consist of double and multiple stars for which a 5-parameter solution could be applied to each component, usually assuming a common parallax. They basically include all resolved objects ( $\rho \gtrsim 0.1$ arcsec, $\Delta m \lesssim 4 \mathrm{mag})$.

Acceleration Solutions $(G)$ : Binaries with separations less than about 0.1 arcsec were not resolved by Hipparcos. A binary star could still be detected through a curved motion of the photocentre. For such systems, two or four terms were added to the standard 5-parameter model, representing quadratic or cubic polynomials of time in each coordinate $(\alpha, \delta)$.

Orbital Solutions (O): These objects have solutions for the seven Keplerian elements of the orbit of the photocentre around the centre of mass, in addition to the five astrometric parameters for the centre of mass.

VIM Solutions (V): Variability-Induced-Movers are unresolved systems, in which non-linear motions of the photocentre are induced by variability. (Not considered in this study)

Stochastic Solutions $(X)$ : These represent about 1 per cent of the Hipparcos objects for which good solution could be found. Since the standard 5-parameter fit gave too large residuals to be explained by observational errors, some sort of multiplicity is probably implied.

Delta-mu binaries $(\Delta \mu)$ : Found by comparing quasi-instantaneous proper motions measured by Hipparcos with long-term averaged, ground-based proper motions (Wielen et al. 1999).

Dividing our sample of main-sequence survey stars into these different categories we find

$$
\begin{array}{lll}
N=13092 & G_{2}=428 & O=112 \\
C=1969 & G_{3}=144 & X=126,
\end{array}
$$

where $N$ is the total sample size and $G_{2}, G_{3}$ are the number of quadratic and cubic acceleration solutions. 


\section{Method}

A non-dynamical galactic model is used to create a synthetic catalogue of main sequence single stars. The scale heights and luminosity functions are based on Hipparcos observations (Holmberg et al. 1997). Interstellar extinction is based on Hakkila et al. (1997). Since we only consider main-sequence stars, a single mass-luminosity relation can be used, which is based on Andersen (1991) for masses above $0.59 \mathcal{M}_{\odot}$ and on Chabrier et al. (1996) for masses down to $0.06 \mathcal{M}_{\odot}$.

To determine the detection probabilities, all stars in the synthetic catalogue are assumed to have a companion. The distributions of orbital parameters are based on DM. In particular, circular orbits are assumed for semi-major axis $a<0.1 \mathrm{AU}$. For $a>2 \mathrm{AU}$, the assumed distribution is $f(e) \propto e$. In the intermediate range, $f(e) \propto e(1-e)^{3}$ is assumed.

The mass of the primary was determined by the mass-luminosity relation described above. The mass of the companion was is found by assuming one of four different mass ratio distributions: a linearly increasing density function $f_{I}(q)=2 q$, a linearly decreasing function $f_{D}(q)=2(1-q)$, a parabolic function $f_{P}(q)=6 q(1-q)$ and a distribution where the companion is a white dwarf. These four functions can be superimposed in any number of ways. For example, the DM distribution (their Fig. 10) is reasonably approximated by $0.1 f_{I}+0.9 f_{D}$, at least for $q>0.1$.

The individual masses and semi-major axis determine the orbital period $(P)$ through Kepler's third law. The time of periastron passage was then randomly chosen between 0 and $P$, and the orbital plane was randomly oriented in space. Since the distance to the object is known from the Galaxy model, the component positions in the apparent orbits on the sky plane could be calculated for each observational epoch defined by the Hipparcos observational strategy, the scanning law.

The observational scanning law and a similar, but simplified, data reduction scheme used for the true Hipparcos observations are applied to each synthetic binary system. This consisted of a series of tests where once a test was passed, the binary was considered detected and no further tests were given. A detailed description of this work can be found in Quist and Lindegren (2000).

\section{Results}

The range of semi-major axes studied was $a=10^{-2}$ to $10^{5} \mathrm{AU}$. Separate synthetic catalogues were generated for each decade in $a$ and for the four basic mass-ratio distributions $\left(f_{I}, f_{D}, f_{P}, f_{W}\right)$. The expected number of binary solutions as a function of the semi-major axis and mass ratio distribution were then compared to the numbers given in Equation 2.

We then estimate the semi-major axis distribution is three ways. The first was to assume that the distribution of $a$ is log-normal with the same width as found by $\mathrm{DM}\left(\sigma_{\log P}=2.3\right.$, corresponding to $\left.\sigma_{\log a}=1.53\right)$. It should be noted that the increasing, parabolic and uniform $\left(0.5 f_{I}+0.5 f_{D}\right)$ mass ratio distributions all gave unacceptable results $\left(\chi^{2}>20\right.$ with 4 degrees of freedom). On the other hand, the decreasing distribution $\left(f_{D}\right.$, having $0 \%$ or $10 \%$ white 
dwarf companions) gave reasonable fits. These are shown in Fig 1 as the heavy and thin solid lines, respectively. The DM distribution is shown for comparison.

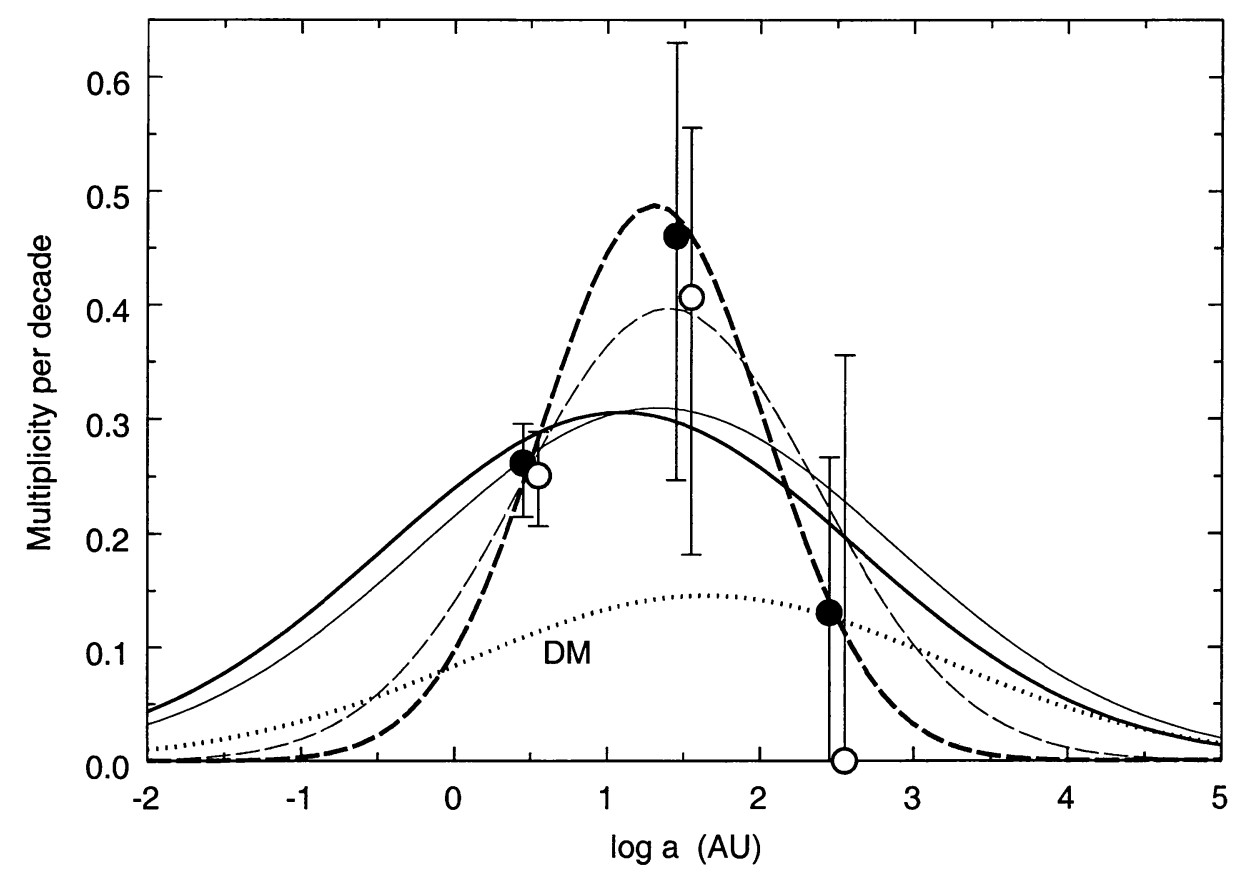

Figure 1. The distribution of semi-major axis determined in three ways. The solid lines have the same log normal width as DM. The dashed lines fit a log normal curve without the constraint on width. The discrete point per decade are from direct inversion and the $95 \%$ confidence intervals are shown. The heavy lines and filled circles assume no white dwarfs companions while the thin lines and open circles assume $10 \%$. The DM distribution is shown for comparison.

The constraint on the width of the distribution was then removed and then fit to the observations, again assuming a decreasing mass ratio distribution and having either $0 \%$ or $10 \%$ white dwarf companions. These are plotted in Fig 1 as heavy $(0 \%)$ and thin $(10 \%)$ dashed lines.

Finally, we attempt to estimate the distribution by direct inversion of the observed counts by a least squares method. These are plotted by filled $(0 \%$ white dwarfs) and open ( $10 \%$ white dwarfs) circles. The associated $95 \%$ confidence intervals were also calculated. In the 1-10 AU separation range, we find $0.25 \pm$ 0.05 , while in the $10-100 \mathrm{AU}$ range we find $0.43 \pm 0.20$ companions per primary. Some point are not shown because the calculated errors were larger than the ordinate range. Note, that the errors in the 1-10 AU range have the smallest confidence intervals, and that the different estimates agree very well in that range.

Extrapolating to the full range of $\log a$, we find that a binary frequency between 0.9 and 1.2 companions per primary is needed to reproduce these results, 
compared to DM's 0.57. This difference may be caused by the different stellar samples, suggesting an increased multiplicity for earlier spectral types.

\section{References}

Andersen, J. 1991, A\&AR, 3, 91

Blaauw, A. 1981, in Scientific importance of high angular resolution, ed. M. H. Ulrich \& K. Kjär, ESO Conference Proceedings, ES0, Garching, 391

Chabrier, G., Baraffe, I., Plez, B. 1996, ApJ, 459, L91

Duquennoy, A., Mayor, M. 1991, A\&A, 248, 485

ESA 1997, The Hipparcos and Tycho Catalogues, ESA SP-1200

Hakkila, J., Myers, J. M., Stidham, B. J., Hartmann, D. H. 1997, AJ, 114, 2043

Holmberg, J., Flynn, C., Lindegren, L. 1997, in Hipparcos Venice 97, ESA SP402, ed. M. A. C. Perryman \& P. L. Bernacca, ESA, Noordwijk, 721

Quist, C. F., Lindegren, L. 2000, A\&A, 361, 770

Turon, C., Crézé, M., Egret, D., et al. 1992, The Hipparcos Input Catalogue, ESA SP-1136

Wielen, R., Dettbarn, C., Jahreiß, H., Lenhardt, H., Schwan, H. 1999, A\&A, 346,675

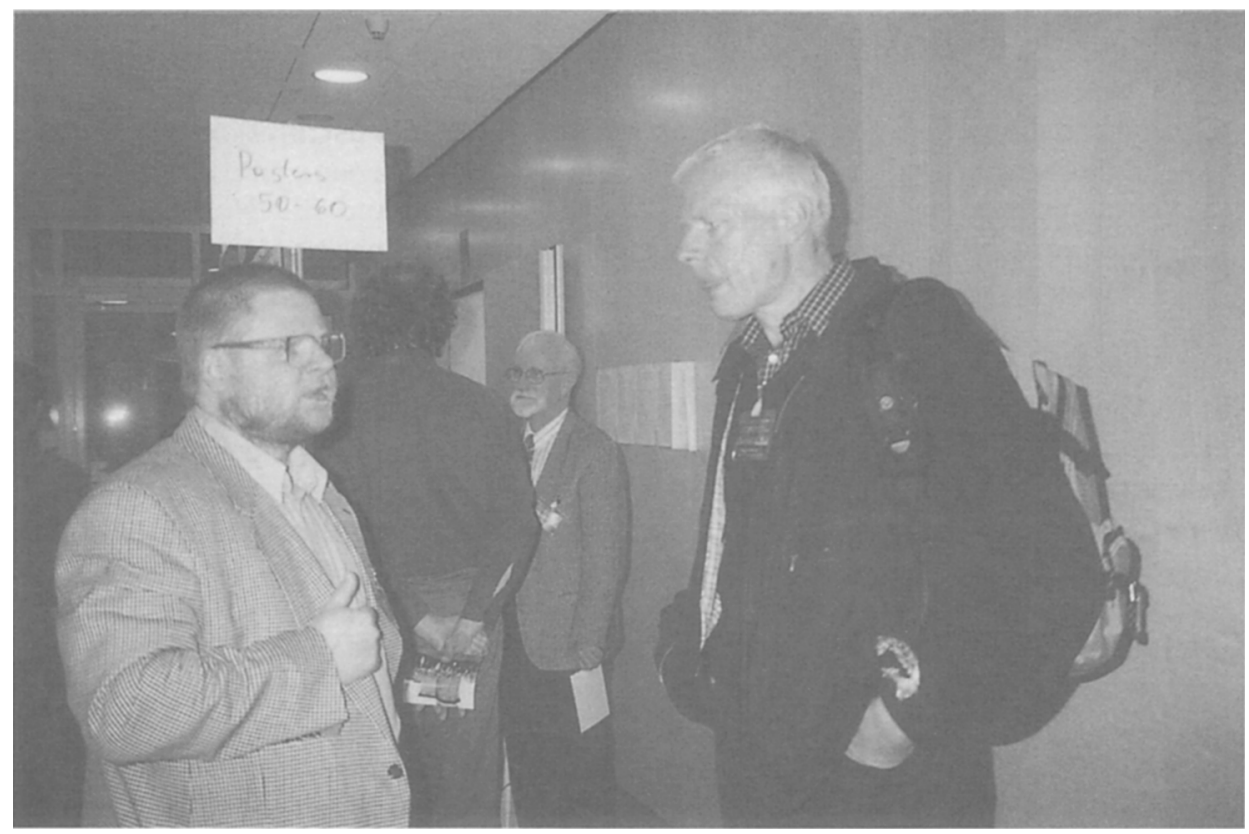

Quist's friends: Ralf-Dieter Scholz and Hartmut Jahreiss 